\title{
The relationship between operating maintenance and lightning overvoltage in distribution networks based on PSCAD/EMTDC
}

\author{
Xiaojun Chen ${ }^{\mathrm{a}}{ }^{*}$, Wenjie Zheng ${ }^{\mathrm{b}}$, Shu Huang ${ }^{\mathrm{c}}$, Hui Chen ${ }^{\mathrm{d}}$ \\ Electric Power Research Institute of Guangdong Power Grid Corporation \\ Limited,Guangzhou,Guangdong 510080, China \\ a chenarmy11@qq.com, b zhengwenjie@gddky.csg.cn, c huangshu@gddky.csg.cn, \\ dchenhui@gddky.csg.cn
}

Corresponding Author. * chenarmy11@qq.com

\begin{abstract}
Keywords: Operating maintenance; Lightning overvoltage; Distribution networks; Overhead line.
Abstract. The model of lighting direct strokes on $10 \mathrm{kV}$ distribution line in Guangzhou was built and the transient procedure was considered based on the software of PSCAD/ECTDC. The effect of arrest and ground resistance operating maintenance on lightning overvoltage in distribution networks has been discussed. The results show that operating maintenance of arrest has significant suppression on lightning overvoltage. In addition, the ground resistance of tower has little influence on the lightning overvoltage.
\end{abstract}

\section{Introduction}

Electric power distribution system constitute the greatest risk to the interruption of power supply. However, power supply in distribution system draws less attention than that in generation and transmission system before. Recently, with the development of economy, especially high-technology, distribution network, an important component of power system has drawn more and more attention. Lightning overvoltage is the major cause of line insulation flashovers in distribution system. Therefore, there are a large number of researches on experimental and theoretical calculation by investigating lightning overvoltage on the overvoltage in distribution networks [1-6].

Lightning overvoltage in power system can be divided into four types: (i) direct strokes, (ii) direct strokes to nearby structures, (iii) surge transfer from medium-voltage (MV) to LV voltage lines through distribution transformers, or (iv) induced voltages due to nearby strokes. (i) and (iv) is the main strokes in low distribution power system. Concerning low voltage overhead lines, the main protective measures against short interruptions and voltage sags originated by lightning-induced voltages can be identified as (i) use of surge arresters and/or (ii) use of shielding wires, (iii) increasing of the line insulation level. In this paper, we will focus on (i).

The operating maintenance in power distribution system is of vital important for overvoltage by lightning strokes $[7,8]$. Operating maintenance can improve the reliability of electrical equipment. The operating maintenance in distribution system is related to the arrest and ground resistance.

The objective of the present paper is the analysis of the effectiveness of operating maintenance (arrest and ground resistance) on lightning overvoltage in distribution networks for a given $10 \mathrm{kV}$ system. The model of lighting direct strokes on $10 \mathrm{kV}$ distribution line in Guangzhou was built and the transient procedure was considered based on the software of PSCAD/ECTDC.

\section{System modeling}

The study was performed using a real $10 \mathrm{kV}$ power system with the configuration shown in Figure 1. The digital simulations were made using PSCAD/EMTDC software. PSCAD was first conceptualized in 1988 and began its long evolution as a tool to generate data files for the EMTDC simulation program. PSCAD (Power Systems CAD) is a powerful and flexible graphical user interface to the world-renowned, EMTDC solution engine. EMTDC is most suitable for simulating the time 
domain instantaneous responses (also popularly known as electromagnetic transients) of electrical systems [9].

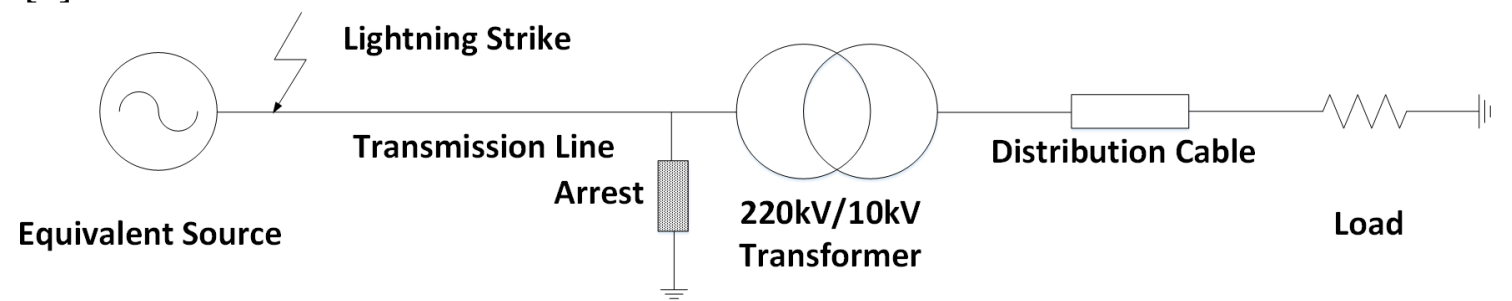

\section{lightning current model}

Figure $1 \mathrm{~A}$ real $10 \mathrm{kV}$ power system simulated in this study

The waveform of the lightning current is set as standard 2.6/50 $\mu$ s double-exponential impulse function [10]

$$
i(t)=A I_{m}\left(e^{-\alpha t}-e^{-\beta t}\right)
$$

Where $A=1.058, \alpha=15000, \beta=1860000$.

The peak value of waveform is set as $10 \mathrm{kA}$ and the impedance of the lightning channel is $300 \Omega$. The lightning current in this paper is negative due to the fact that the negative lightning current is more usual than positive. Figure 2 illustrates I-t characteristic curve of arrester.

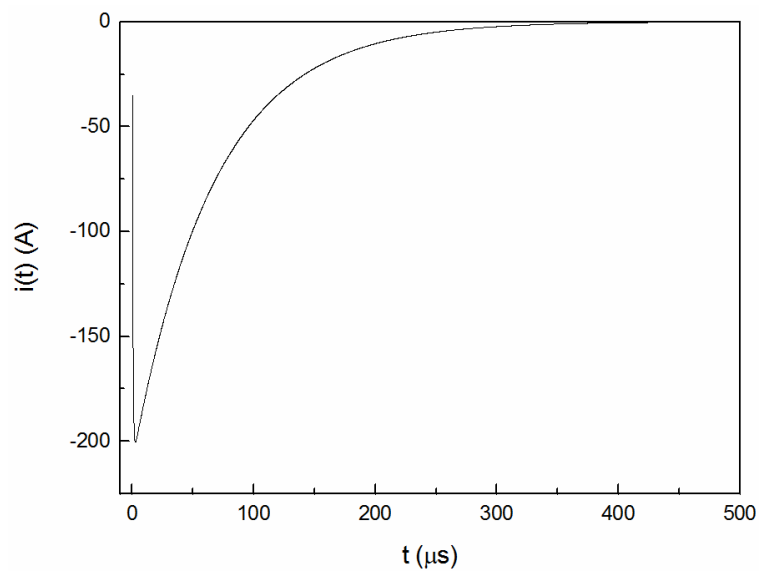

Figure 2 I-t characteristic curve of lightning current

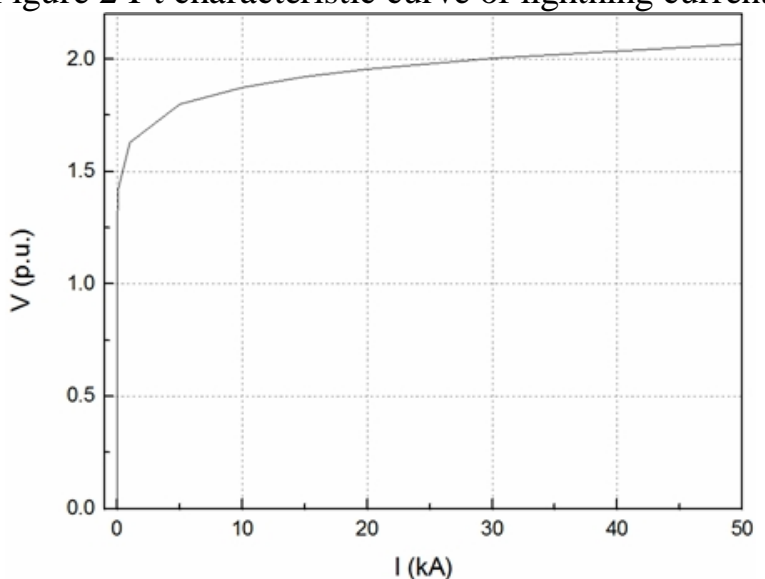

Figure 3 Non-linear V-I characteristic curve of arrester

\section{Surge arrester model}

Metal oxide arresters, which present a highly non-linear voltage-current characteristic, are significant for overvoltage coordination studies involving fast front surges. The metal oxide surge arrestor is modeled as a non-linear resistor in series with a variable voltage source. The V-I characteristic of $10 \mathrm{kV}$ rated arrester each nonlinear resistor is illustrated in Figure 3.

\section{Line model}

The transmission lines and cables are modeled using one of three distributed (travelling wave) models: Bergeron, frequency dependent (mode), frequency dependent (phase). The most accurate of these is the frequency dependent (Phase) model, which represents all frequency dependent effects of a 
transmission line, and should be used whenever in doubt. The transmission line was considered to be ideally transposed and the frequency dependence of the longitudinal parameters was modeled. The distribution cable adopts the Bergeron mode. The impedance of distribution cable is $40 \Omega$.

\section{Transformer model}

There are two primary methods by which transformers are modeled in PSCAD: The Classical and the Unified Magnetic Equivalent Circuit (UMEC) methods. In order to analyze the lightning response, transformer modeling for lightning surge analysis considering a capacitance set. The model of transformer considering capacity in Figure 4.

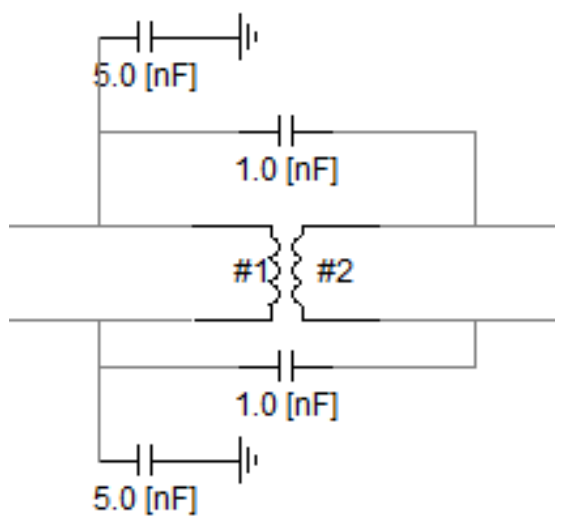

Figure 4 Model of transformer considering capacity

\section{Results and analysis}

\section{The relationship between overvoltage and arresters}

In this case, the ground resistance of tower is fixed to $5 \Omega$ and the lightning current strokes the lightning conductor because the back striking has higher probability than the lightning shielding failure. In addition, the arresters are installed in the high voltage side of transformer.

The position of the lightning current can be divided into near and far from transformer. Figure 5 show the influence of arresters on overvoltage. From the Figure 5a, it can be concluded that the maximum overvoltage $(300 \mathrm{kV})$ takes place in the phase B when the lightning strike at far tower with arrest. Although the maximum value also comes from the same phase when the lightning strokes at near tower with arrest (Figure 5b), it decreases more compared with the lightning striking at the far tower. Therefore, the lighting position has a great influence on the lightning overvoltage.

Figure 5c and Figure 5d show that the great overvoltage will occur when the arrests break down. Compare the case including the arrest, the maximum overvoltage originate from phase A. Besides, the overvoltage when the lightning strokes far tower without arrester is smaller than that when the lightning strike near tower, which is contrast with the case including arrest.

In conclusion, the operating maintenance of the arrest is of great importance for the safe operation of power distribution system. Field operator should check up and maintain the arrests regularly.

\section{The relationship between overvoltage and ground resistance of tower}

In this case, the arresters are installed in the primary side of transformer and the ground resistance of transformer substation is $2 \Omega$. 

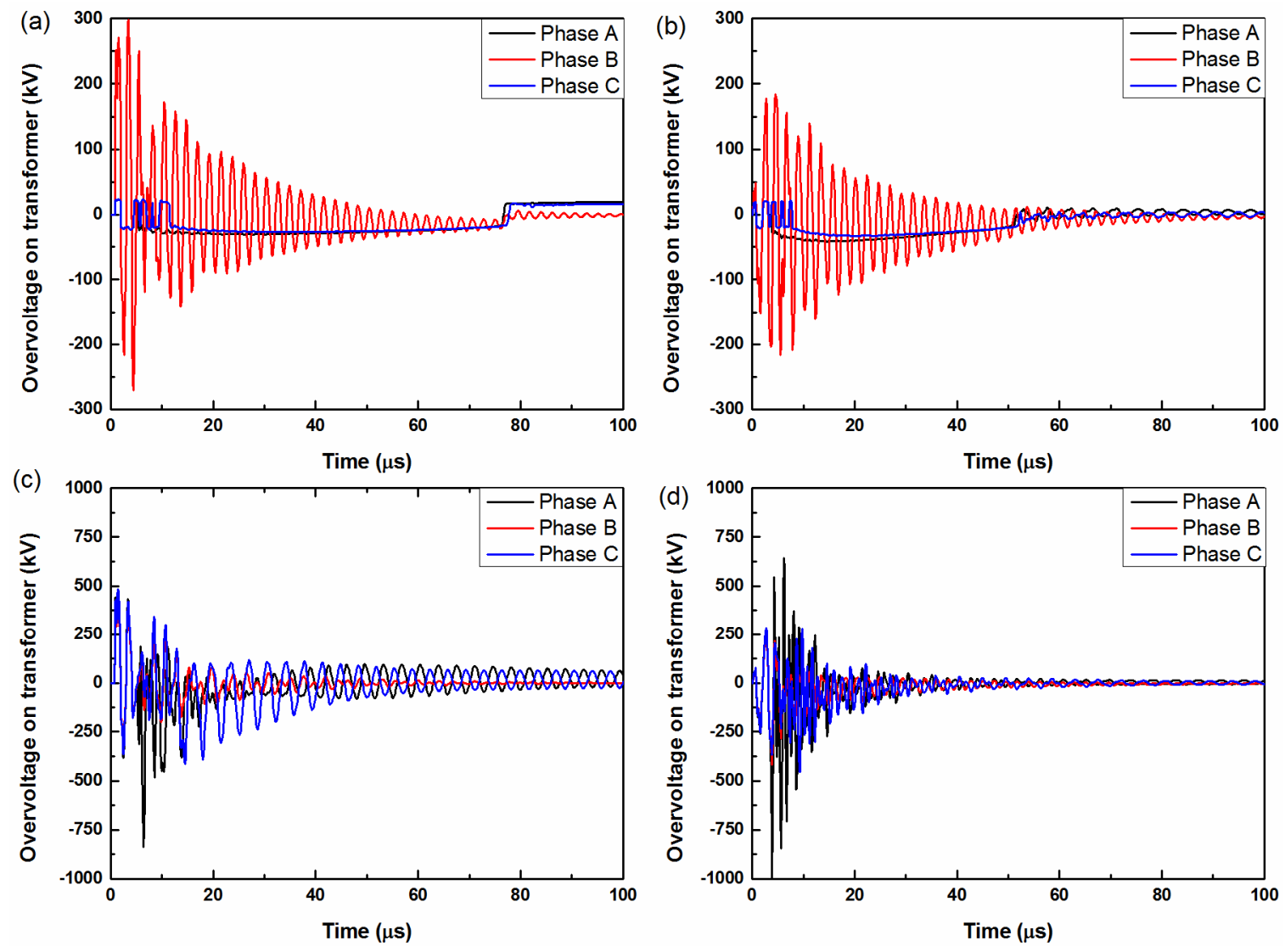

Figure 5 Simulation results of overvoltage. (a) Lightning strokes at far tower with arrest; (a) Lightning strokes at near tower with arrest; (a) Lightning strokes at far tower without arrest; (a) Lightning strokes at near tower without arrest.
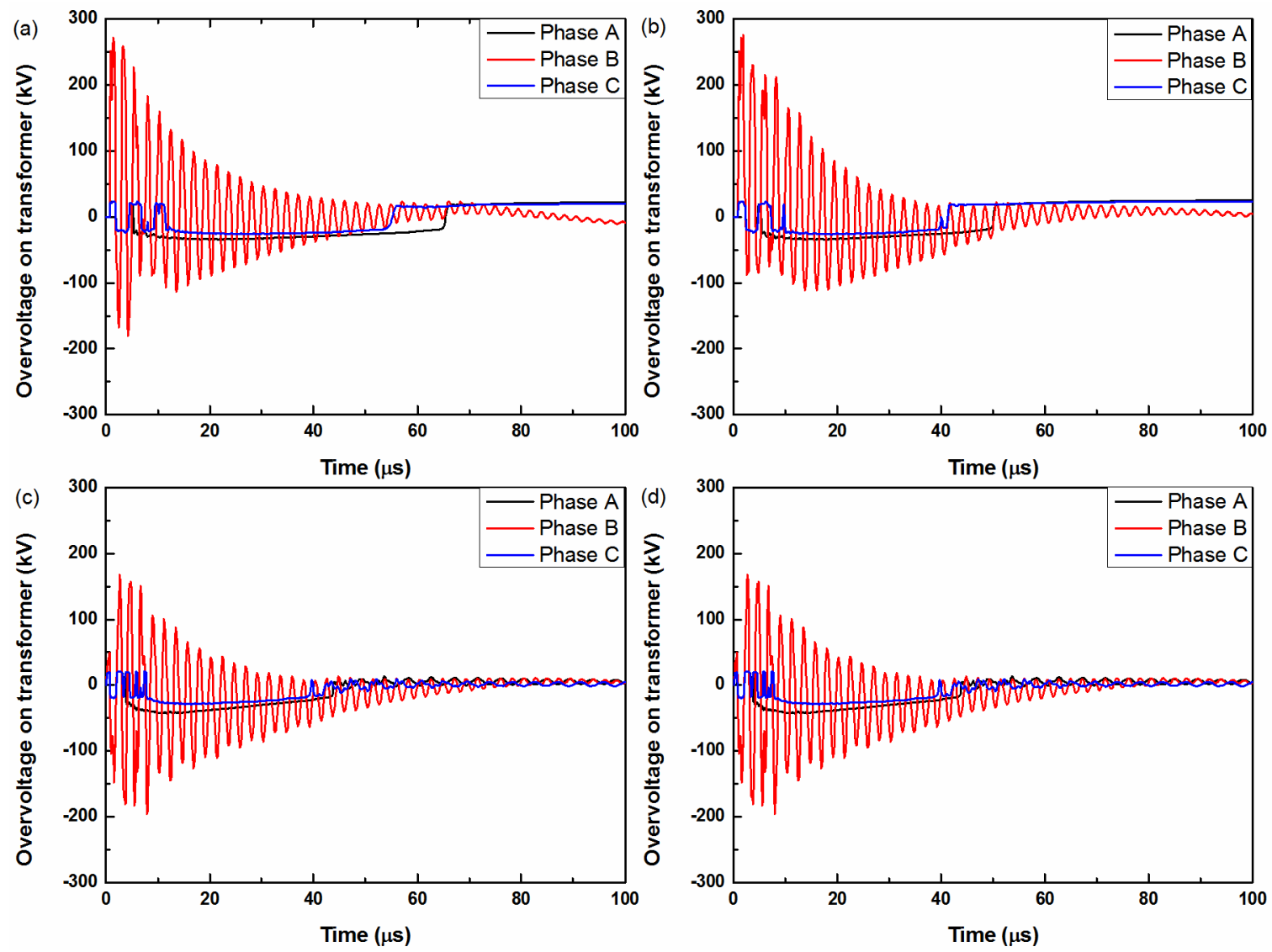

Figure 6 Simulation results of overvoltage. (a) Lightning strokes at far tower with $10 \Omega$ ground resistance; (b) Lightning strokes at far tower with $20 \Omega$ ground resistance; (c) Lightning strokes at near tower with $10 \Omega$ ground resistance; (d) Lightning strokes at near tower with $20 \Omega$ ground resistance. 
Figure 6 presents the overvoltage change from the ground resistance of tower. It can be seen that phase $\mathrm{B}$ has the maximum overvoltage when the arresters are correctly configured. With the increase of the ground resistance of tower, the overvoltage maintains the same value. In addition, the value goes down when the lightning strokes form far tower to near tower, in accordance with the results shown in 3.1 .

\section{Conclusion}

In summary, the model of lighting direct strokes on $10 \mathrm{kV}$ distribution line in Guangzhou was built in PSCAD/EMTDC. The results show than operating maintenance of arrest has an important effect on the overvoltage induced by lightning current.

\section{Acknowledgement}

This work was supported in part by science and technology program of China Southern Power Grid under grant K-GD2013-0482.

\section{References}

[1] A.D. Conti, F.H. Silveira, S. Visacro. Lightning overvoltages on complex low-voltage distribution networks. Electric Power Systems Research 85 (2012) 7-15.

[2] S. Sekioka, K. Aiba, S. Okabe. Lightning overvoltages on low voltage circuit caused by ground potential rise. International Conference on Power Systems Transients, Lion, France. 2007.

[3] C.A. Nucci, F. Rachidi, et al. Lightning-induced voltages on overhead lines. IEEE Transactions on Electromagnetic Compatibility 35 (1993) 75-86.

[4] I. Metwally, F.H. Heidler. Computation of transient overvoltages in low-voltage installations during direct strokes to different lightning protection systems. IEEE Transactions on Electromagnetic Compatibility 49 (2007) 602-613.

[5] P. Hasse. Overvoltage protection of low-voltage systems. IET, London, 2000.

[6] A.D. Conti, S. Visacro. Evaluation of lightning surges transferred from medium voltage to low-voltage networks. IEE Proceedings-Generation, Transmission and Distribution 152 (2005) 351-356.

[7] L. Bertling, R. Allan, and R. Eriksson. A reliability-centered asset maintenance method for assessing the impact of maintenance in power distribution systems. IEEE Transactions on Power Systems, 20 (2005) 75-82.

[8] P. Hilber. Maintenance optimization for power distribution systems. (2008).

[9] PSCAD®/EMTDCTM vs 4.2 User'Guide, Manitoba HVDC Research Centre (2005).

[10] F. Heidler, J. M. Cvetić, B. V. Stanić. Calculation of lightning current parameters. IEEE Transactions on Power Delivery, 14 (1999) 399-404. 